EPJ Web of Conferences 81, 01001 (2014)

DOI: 10.1051/epjconf/ 20148101001

(C) Owned by the authors, published by EDP Sciences, 2014

\title{
Testing the Standard Model at the precision frontier: the anomalous magnetic moment of the muon
}

\author{
Andreas Hafner ${ }^{1, a}$ \\ ${ }^{1}$ Institut für Kernphysik, Johannes Gutenberg-Universität Mainz, Germany
}

\begin{abstract}
The anomalous magnetic moment of the muon $(g-2)_{\mu}$ is one of the most precisely measured quantities in particle physics $(0.54 \mathrm{ppm})$. There is a long-standing discrepancy of 3-4 standard deviations between the direct measurement of $(g-2)_{\mu}$ and its theoretical evaluation.

This theoretical prediction is subdivided into three contributions: QED, weak and hadronic. The QED and weak parts can be determined in perturbative approaches with very high precision. Thus, the hadronic uncertainty dominates the total theoretical uncertainty. Within the hadronic uncertainty, the largest contribution stems from the vacuum polarization term, which can be evaluated with the measurement of the inclusive hadronic cross section in $e^{+} e^{-}$annihilation. The second largest contribution to the hadronic uncertainty stems from the so-called Light-by-Light amplitudes. They have to be evaluated via theoretical models. These models require transition form factor measurements as input. Existing and future measurements of the relevant hadronic cross sections and transition form factors are presented.
\end{abstract}

\section{Introduction}

There are various ways to test the Standard Model (SM) of particle physics. Many approaches include searches for new particles or phenomena at the high energy frontier. Another approach is to measure SM observables with high precision and compare the measurement to the SM prediction. One particular test at this high precision frontier is the measurement of the anomalous magnetic moment of the muon $a_{\mu}=0.5 \cdot(g-2)_{\mu}$. On the one hand, it is one of the most precisely measured observables in particle physics. On the other hand, there is a discrepancy of 3-4 standard deviations between the direct measurement of $(g-2)_{\mu}$ and its theoretical evaluation [1,2].

This theoretical prediction is subdivided into three contributions: QED, weak and hadronic. The QED and weak parts can be determined in perturbative approaches with very high precision [3]. At low energies perturbation theory cannot be used to calculate the hadronic contribution $a_{\mu}^{\text {had }}$. Thus, the hadronic uncertainty dominates the total theoretical uncertainty. Within the hadronic uncertainty, the largest contribution stems from the vacuum polarization (VP) term. It is possible to relate this contribution $a_{\mu}^{\text {had,VP }}$ via a dispersion relation (Eq. (1)) to hadronic cross sections, which typically are

ae-mail: hafner@kph.uni-mainz.de 
measured in $e^{+} e^{-}$energy scan experiments at low energies.

$$
a_{\mu}^{\text {had }, L O}=\frac{1}{4 \pi^{3}} \int_{m_{\pi^{o}}^{2}}^{\infty} d s K(s) \sigma_{e^{+} e^{-} \rightarrow \text { hadrons }}(s) .
$$

The Kernel function $K(s)$ is proportional to $s^{-1}$ [4]. As an alternative to the energy scan experiments, the study of the Initial State Radiation (ISR) events at flavor-factories allows independent measurements of exclusive hadronic cross sections. This method allows high statistics $e^{+} e^{-}$experiments running at a fixed center-of-mass (c.m.) energy to access processes at lower effective c.m. energies by studying events with a high energy photon emitted from the initial state. The use of this technique at high luminosity $\phi$ - and $B$-factories has been discussed in detail in Refs. [5-7].

The second largest contribution to the hadronic uncertainty stems from the so-called hadronic Light-by-Light (LbL) amplitudes. They have to be evaluated via theoretical models. These models require transition form factor measurements as input.

Existing and future measurements of $(g-2)_{\mu}$, the relevant hadronic cross sections, and transition form factors are presented.

\section{Experiments}

\section{$2.1(g-2)_{\mu}$ at BNL}

The most precise measurement of $a_{\mu}=(11659208.9 \pm 6.4) \cdot 10^{-10}$ has been performed at BNL [8]. The production of the polarized muon beam is illustrated in Fig. 1 (left). It is essential that the decay of $\pi^{+} \rightarrow \mu^{+} v$ violates parity, which leads to longitudinal polarization of the muons.
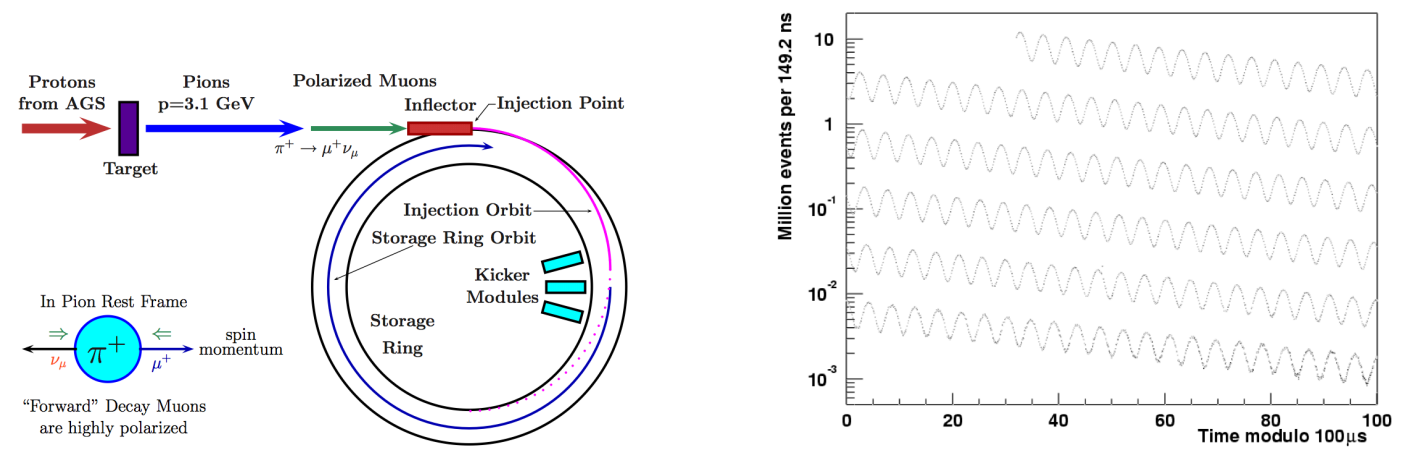

Figure 1. Illustration of muon production, injection and storage in the BNL $(g-2)_{\mu}$ ring (left) and the electron detection rate (right) [8].

These muons are guided into a storage ring with constant magnetic field $\vec{B}$, and thus perform a rotating motion with cyclotron frequency $\vec{\omega}_{c}$. The spin of the muons is precessing with the frequency $\vec{\omega}_{s}$. This leads to a change of the helicity with the Lamor frequency $\vec{\omega}_{a}$ (Eq. 2).

$$
\vec{\omega}_{a}=\vec{\omega}_{s}-\vec{\omega}_{c}=-\frac{q}{m}\left[a_{\mu} \vec{B}-\left(a_{\mu}-\frac{1}{\gamma^{2}-1}\right) \frac{\vec{\beta} \times \vec{E}}{c}\right]=-a_{\mu} \frac{q}{m_{\mu}} \vec{B}
$$

The last equality of Eq. 2 is true for a particular choice of the so-called magical muon energy $E_{\text {magic }}=$ $3.098 \mathrm{GeV}$. Finally the decay of $\mu^{+} \rightarrow e^{+} \bar{v} v$ again violates parity. Thus, the $e^{+}$direction remembers 
the $\mu^{+}$polarization and the number of detected $e^{+}$above an energy threshold is modulated with $\vec{\omega}_{a}$, see Fig. 1 (right). This allows the extraction of $a_{\mu}$. Future experiments at Fermilab [9] and JPARC [10] intend to further improve the precision by a factor of 4 .

\subsection{Meson-Photon transition form factors}

The uncertainty of the hadronic LbL contribution [11], $\Delta a_{\mu}^{\text {had,LbL }}=2.6 \cdot 10^{-10}$, to the theoretical estimate of $(g-2)_{\mu}$ is larger than the expected uncertainties of the upcoming direct $(g-2)_{\mu}$ measurements, $\sim 1.5 \cdot 10^{-10}$. The LbL estimates can be improved with experimental input, especially from photon-meson transition form factors (TFF). Here, the contribution of the $\pi^{0} \mathrm{TFF}$ is dominating.

\subsection{1 $\pi^{0}$-photon transition form factor}

Measurements of the $\pi^{0}$ TFF are shown in Fig. 2 (left) [12-15]. BABAR agrees with CLEO in the region $4 \mathrm{GeV}^{2}<Q^{2}<9 \mathrm{GeV}^{2}$. However, it clearly exceeds the expected asymptotic limit by pQCD: $\lim _{Q^{2} \rightarrow \infty} F\left(Q^{2}\right)=\sqrt{2} f_{\pi}$, taking into account systematic uncertainties of the efficiency (2.5\%), background $(0.3-6.0 \%)$, mostly due to $e^{+} e^{-} \rightarrow e^{+} e^{-} \pi^{0} \pi^{0}$, and additional model uncertainty $(1.5 \%)$. The recent measurement by Belle with similar systematic uncertainties is in disagreement with $B A B A R$ at large $Q^{2}$. The slope is in better agreement with pQCD, but also exceeds the asymptotic prediction.
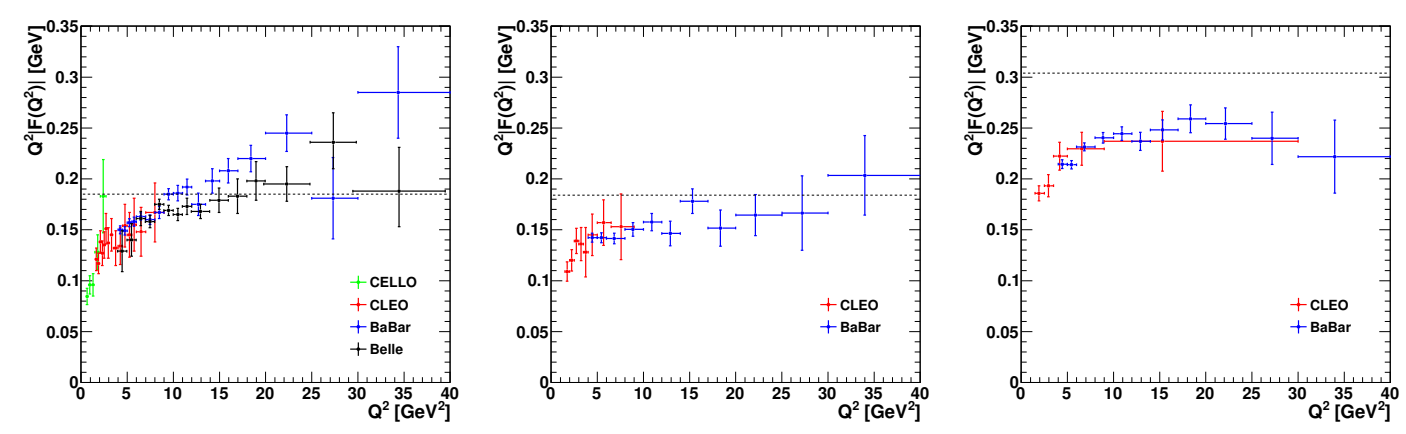

Figure 2. Meson-Photon TFFs for the $\pi^{0}$ (left), $\eta$ (middle), and $\eta^{\prime}$ (right) mesons of various experiments [12-14]. The dashed lines represent the asymptotic limit expected by pQCD.

\subsection{2 $\eta / \eta^{\prime}$-photon transition form factors}

The $\eta$ TFF measurement as a function of momentum transfer $Q^{2}$ from BABAR [12] and CLEO [15] is shown in Fig. 2 (middle). For BABAR the systematic uncertainty is $2.9 \%$, dominated by model and $\pi^{0}$ reconstruction uncertainties of the $\eta \pi^{+} \pi^{-} \pi^{0}$ final state

The $\eta^{\prime} \rightarrow \pi^{+} \pi^{-} \eta$, with $\eta \rightarrow \gamma \gamma$ measurements are shown in Fig. 2 (right). The BABAR systematic uncertainty of $3.5 \%$ is dominated by model and $\eta$ reconstruction uncertainties. The $\eta^{\prime}$-TFF stays below the asymptotic expectation.

More data is needed in order to solve this puzzling situation for the TFFs at large $Q^{2}$ and as input for $a_{\mu}^{\text {had,LbL }}$ at small $Q^{2}$, especially for the $\pi^{0}$ final state. 


\subsection{Hadronic cross sections}

The dominant contribution to the hadronic part of $(g-2)_{\mu}$ stems from the VP, requiring hadronic cross section measurements as input. The $e^{+} e^{-} \rightarrow \pi^{+} \pi^{-}$cross section has the largest contribution $(\sim 75 \%)$.

\subsection{1 $\sigma\left(e^{+} e^{-} \rightarrow \pi^{+} \pi^{-}\right)$}

Fig. 3 (left) shows the precise $\sigma$ measurements that have been performed in the $\rho$ peak region. The $\rho$ $\omega$ interference is clearly visible. The relative difference between the two most precise measurements, from $B A B A R$ and KLOE, is shown in Fig. 3 (right). We observe a disagreement of several standard deviations at and above the $\rho$ resonance peak, leading to a large uncertainty of $a_{\mu}$. Additional high precision measurements are expected from BES-III, CMD-III and SND in the near future.
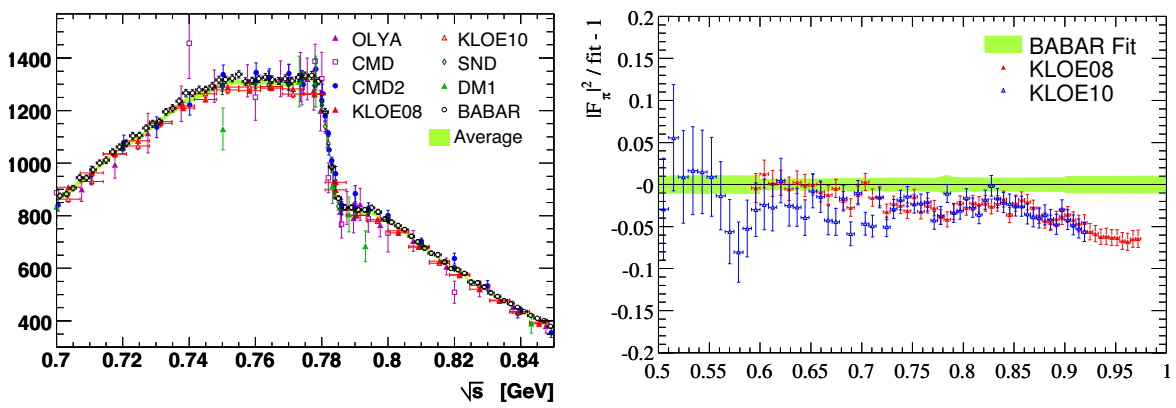

Figure 3. Left: cross section of $e^{+} e^{-} \rightarrow \pi^{+} \pi^{-}$in the $\rho$-peak region from OLYA [16], CMD [17], SND [18], DM1 [19], KLOE [20], and BABAR [21]. Right: relative difference between BABAR and KLOE.

\subsection{2 $\sigma\left(e^{+} e^{-} \rightarrow K \bar{K}\right)$}

Recently, the $e^{+} e^{-} \rightarrow K^{+} K^{-}$final state has been published by BABAR [21], see Fig. 4. In the $\phi$ peak region, this measurement is in agreement within 2 standard deviations to the CMD2 [22] and SND [23] data. At larger cms energies (right) a different shape in comparison to SND is observed.
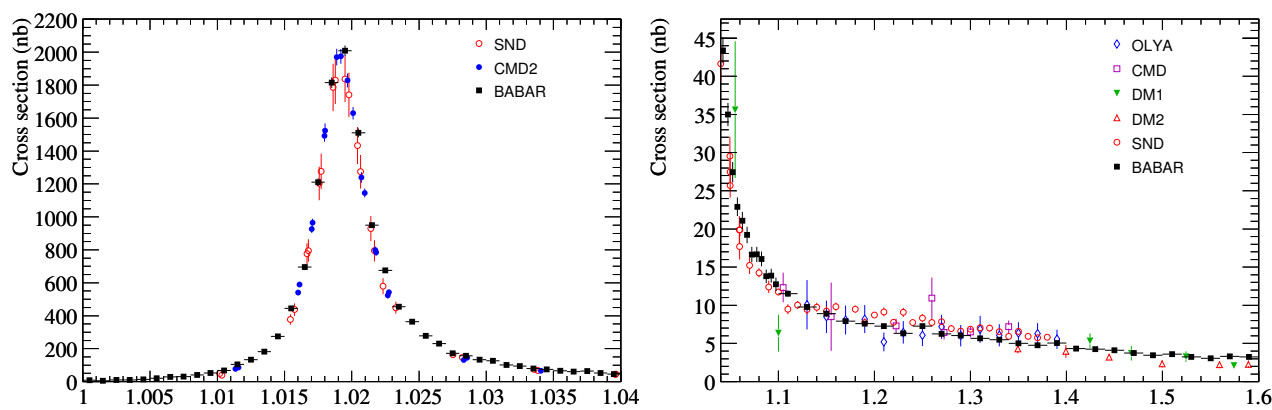

Figure 4. Cross section of $e^{+} e^{-} \rightarrow K^{+} K^{-}$in (left) and above (right) the $\phi$ peak region. 
In Fig. 5 (left), the invariant $K_{S}^{0} K_{L}^{0}$-mass distribution by the $B A B A R$ [24] experiment in the $\phi$ peak region is shown, from which the partial electronic width of $\phi$ to $K_{S}^{0} K_{L}^{0}$ is extracted. This observable is in agreement with the CMD2 [25] result. At larger energies, Fig. 5 (right), a clear structure is visible, which might be due to the $\phi(1680)$ resonance.
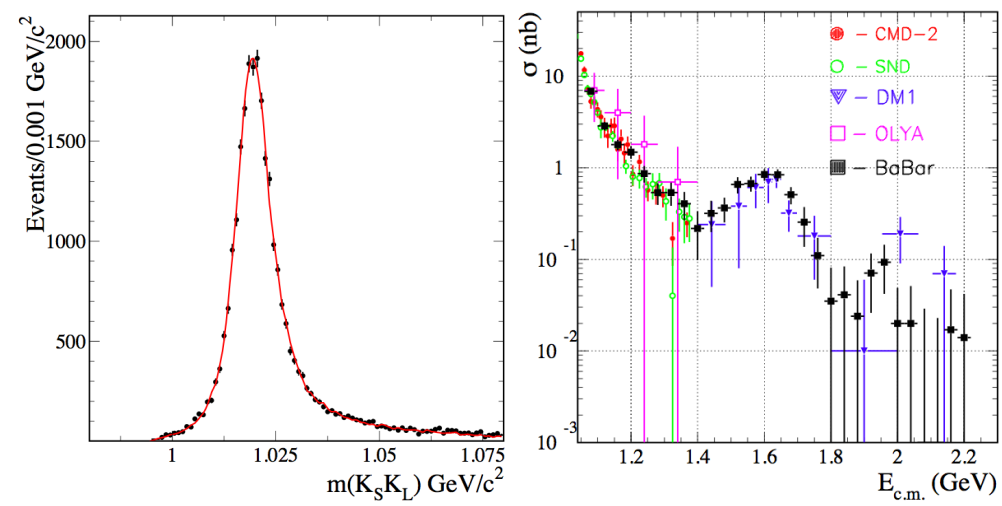

Figure 5. Left: invariant $K_{S}^{0} K_{L}^{0}$-mass distribution by the $B A B A R$ experiment in the $\phi$ region. Right: cross section of $e^{+} e^{-} \rightarrow K_{S}^{0} K_{L}^{0}$ above the $\phi$ region.

\subsubsection{Multihadronic final states}

In Fig. 6, we see the first measurement of the hadronic cross section of the $K_{S}^{0} K_{L}^{0} \pi^{+} \pi^{-}$(left), $K_{S}^{0} K_{S}^{0} \pi^{+} \pi^{-}$ (middle), and $K_{S}^{0} K_{L}^{0} K^{+} K^{-}$(right) final states measured by BABAR. This will allow for the first time the direct extraction of the $(g-2)_{\mu}$ contribution of these final states. Up to date the value has been estimated by means of isospin relations.
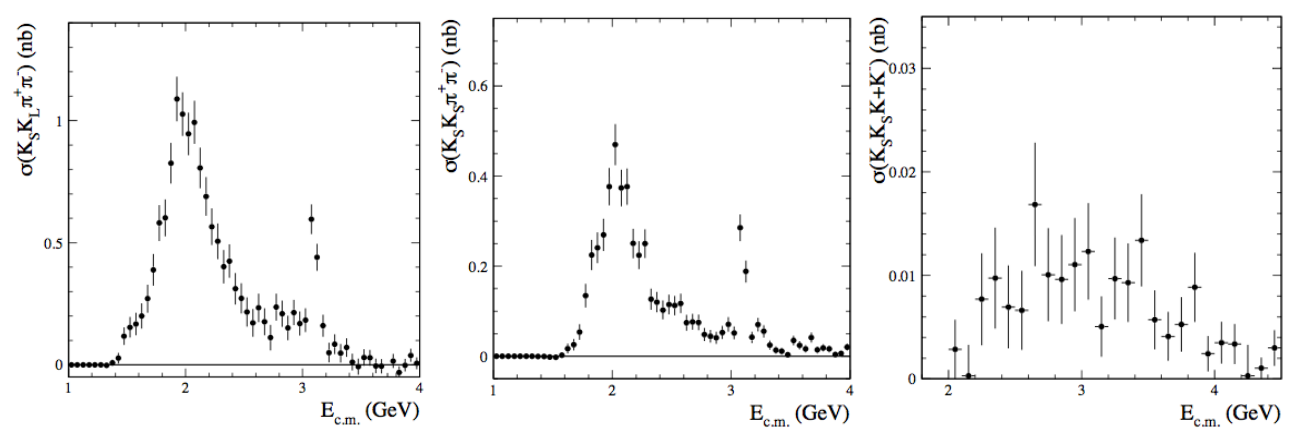

Figure 6. Hadronic cross section of the $K_{S}^{0} K_{L}^{0} \pi^{+} \pi^{-}$(left), $K_{S}^{0} K_{S}^{0} \pi^{+} \pi^{-}$(middle), and $K_{S}^{0} K_{L}^{0} K^{+} K^{-}$(right) final states measured by $B A B A R$. 


\section{Conclusion and outlook}

Including hadronic cross section and TFF measurements the remaining difference between theoretical prediction and measured value of 3.0-3.5 standard deviations [1,2] for the muon anomaly still poses an open question and requires further studies. The measurement of $K \bar{K}\left(\pi^{+} \pi^{-} / K^{+} K^{-}\right)$and await to be included in the world averages. Additional direct $(g-2)_{\mu}$ measurements and hadronic cross section and TFF measurements will help to shed more light in the observed difference.

I want to thank the organizers for the warm hospitality and the organization of this beautiful conference in Cracow.

\section{References}

[1] M. Davier et al., Europ. Phys. J. C 71, 1515 (2011).

[2] T. Teubner et al., Nucl. Phys. Proc. Suppl. 218, 225 (2011).

[3] F. Jegerlehner and A. Nyffeler, Phys. Rept. 477, 1 (2009).

[4] S.J. Brodsky and E. de Rafael, Phys. Rev. 168, 1620 (1968).

[5] A.B. Arbuzov et al., JHEP 9812, 009 (1998).

[6] S. Binner, J.H. Kühn, K. Melnikov, Phys. Lett. B 459, 279 (1999).

[7] M. Benayoun et al., Mod. Phys. Lett. A 14, 2605 (1999).

[8] G.W. Bennett et al. (Muon g-2 Collaboration), Phys. Rev. D 73, 072003 (2006).

[9] G. Venanzoni, (Fermilab E989 Coll.), Nucl. Phys. Proc. Suppl. 225-227, 277-281 (2012).

[10] T. Mibe, (J-PARC g-2 Collaboration), Nucl. Phys. Proc. Suppl. 218, 242-246 (2011).

[11] J. Prades et al., arXiv:0901.0306, (2009).

[12] B. Aubert et al. (BABAR Collaboration), PRD 80, 052002 (2009); PRD 84, 052001 (2011).

[13] S. Uehara et al. (Belle Collaboration), Phys. Rev. D 86, 092007 (2012).

[14] H. J .Behrend et al. (CELLO Collaboration), Z. Phys. C 49, 401 (1991).

[15] J. Gronberg et al. (CLEO Collaboration), Phys. Rev. D 57, 33 (1998).

[16] L.M. Barkov et al. (OLYA, CMD Collaborations), Nucl. Phys. B 256, 365 (1985), I.B. Vasserman et al., (OLYA Collaboration), Sov. J. Nucl. Phys. 30, 519 (1979).

[17] R.R. Akhmetshin et al. (CMD2 Collaboration) Phys. Lett. B 578, 285 (2004); V.M. Aulchenko et al. (CMD2 Collaboration), JETP Lett. 82, 743 (2005); R.R. Akhmetshin et al. (CMD2 Collaboration), JETP Lett. 84, 413 (2006); R.R. Akhmetshin et al. (CMD2 Collaboration), Phys. Lett. B 648, 28 (2007).

[18] M.N. Achasov et al. (SND Collaboration), JETP Lett. 103, 380 (2006).

[19] A. Quenzer et al. (DM1 Collaboration), Phys. Lett. B 76, 512 (1978).

[20] F. Ambrosino et al. (KLOE Collaboration), Phys. Lett. B 670, 285 (2009), Phys. Lett. B 700, 102-110 (2011), Phys. Lett. B 720, 336-343 (2013).

[21] B. Aubert et al. (BABAR Collaboration), PRD 70, 072004 (2004), PRD 71, 052001 (2005), PRD 73, 052003 (2006), PRD 73, 012005 (2006), PRD 76, 092006 (2007), PRD 76, 092005 (2007), PRD 76, 012008 (2007), PRD 77, 092002 (2008), PRL 103, 231801 (2009), PRD 85, 112009 (2012), PRD 86, 032013 (2012), PRD 88, 032013 (2013), PRD 89, 092002 (2014).

[22] R.R. Akhmetshin et al. (CMD Collaboration), Phys. Lett. B 669, 217-222 (2008).

[23] M.N. Achasov et al. (SND Collaboration), Phys. Rev. D 76, 072012 (2007).

[24] J.P. Lees et al. (BABAR Collaboration), PRD 89, 092002 (2014).

[25] R.R. Akhmetshin et al. (CMD-2 Collaboration) Phys. Lett. B 466, 385 (1999), Erratum-ibid. B 508, 217-218 (2001). 